\title{
Hyaluronic acid and oxidized regenerated cellulose prevent adhesion reformation after adhesiolysis in rat models
}

This article was published in the following Dove Press journal:

Drug Design, Development and Therapy

25 October 2016

Number of times this article has been viewed

\section{Yan Zhang \\ Qin Liu \\ Ning Yang \\ Xuegang Zhang}

Department of Gynecology, Kunshan Hospital Affiliated to Jiangsu University, Kunshan, Jiangsu, People's Republic of China
Correspondence: Qin Liu

Department of Gynecology, Kunshan Hospital Affiliated to Jiangsu University, No 91, Qingjin west Road, Kunshan, Jiangsu 215300, People's Republic of China

Tel +86 I39 62676795

Email qinliu@icloud.com
Abstract: Postsurgical adhesion formation is the most common complication in abdominal and pelvic surgery. Adhesiolysis is the most commonly applied treatment for adhesion formation but is often followed by adhesion reformation. Therefore, an efficient strategy should be adopted to solve these problems. This study aimed to explore whether hyaluronic acid and oxidized regenerated cellulose (ORC) could prevent adhesion formation and reformation. Thirty female Sprague Dawley rats were randomly divided into three groups $(\mathrm{n}=10 \mathrm{each})$ and subjected to different treatments during the first and second surgery. The control group was treated with isotonic sodium chloride, the ORC group was treated with ORC $(1.5 \times 1 \mathrm{~cm})$, and the medical sodium hyaluronate (MSH) group was treated with $1 \% \mathrm{MSH}(0.5 \mathrm{~mL})$. At 2 weeks after the first

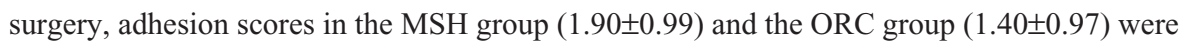

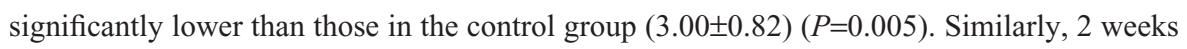

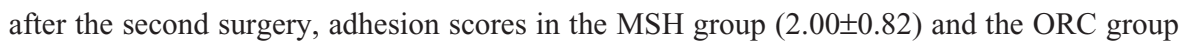

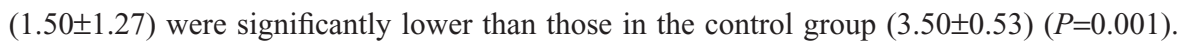
In addition, body weights in the MSH group and the ORC group did not change significantly, whereas the control group showed a consistent decrease in body weight during the experiment. Histological examination revealed that inflammatory infiltration was involved in both adhesion formation and reformation. In conclusion, hyaluronic acid and ORC were both efficient in reducing adhesion formation and reformation in the rat model.

Keywords: hyaluronic acid, oxidized regenerated cellulose, adhesion formation, adhesion reformation, rat model

\section{Introduction}

Postoperative adhesions have become the most frequent complications after abdominal and pelvic surgery, occurring to different degrees in $60 \%-90 \%$ of patients. ${ }^{1}$ This prevalent complication increases the disease burden in affected patients. The characteristic symptoms of postoperative adhesions in $74 \%$ of cases of adhesion-related abdominal obstruction include diffuse or chronic pelvic pain, and more than $40 \%$ of patients develop infertility. ${ }^{2,3}$ In addition, postoperative adhesions result in a large number of reinterventions, increased hospital stays, extended reintervention times, and enormous economic burden. ${ }^{2}$ However, only few specialists are aware of the extent of this serious problem, and increased attention and scientific investigations are urgently required.

Adhesion formation results from the fibrous connections between tissues and organs caused by surgical trauma, inflammation, and endometriosis. ${ }^{4}$ In gynecological surgery, especially in procedures with high risk of adhesion formation, such as ovarian surgery, endometriosis and tubal surgery, and myomectomy, good surgical practice along with 
adoption of adhesion-reducing agents is conducive to preventing adhesion formation. ${ }^{2}$ At present, many anti-adhesion agents, such as oxidized regenerated cellulose (ORC), ${ }^{5}$ hyaluronic acid, ${ }^{6}$ hyaluronate-carboxymethyl cellulose, ${ }^{7}$ and icodextrin, ${ }^{5,8,9}$ are available for use in the clinic. The most frequently adopted treatment approach for postoperative adhesions is adhesiolysis. However, adhesiolysis is often followed by adhesion reformation and relevant studies on managing adhesion reformation are still limited. ${ }^{10}$

The rat uterine horn model is the most frequently used model for investigating postsurgical adhesion formation. ${ }^{11}$ Using this animal model, many effective anti-adhesion agents have been developed, including ORC and sodium hyaluronic acid. ORC was the first synthetic mechanical barrier used for preventing adhesion formation and was designed to be absorbed in the abdominal cavity within 2 weeks. ${ }^{6}$ Sodium hyaluronic acid is a linear polysaccharide with repeating disaccharide units composed of sodium D-glucuronate and $\mathrm{N}$-acetyl-D-glucosamine. ${ }^{6}$ It is a naturally occurring component of many body tissues and fluids and shows lubrication and gliding effects. ${ }^{3}$ The purpose of this experimental study was to explore whether ORC and hyaluronic acid could reduce adhesion reformation in rats.

\section{Materials and methods Animal experiment}

Thirty female Sprague Dawley rats, 3-4 months old and weighing 230-260 g, were used for generating intraperitoneal adhesions in this study. During the entire study period, the animals were housed under controlled conditions of temperature $\left(21^{\circ} \mathrm{C}-22^{\circ} \mathrm{C}\right)$, humidity $(40 \%-60 \%)$, and light (12-hour light/12-hour dark regime), and had free access to food and water. In addition, the animals were starved for 6 hours before the surgery. The study and the study procedures were approved by the Committee on the Ethics of Animal Experiments at the Affiliated Kunshan Hospital of Jiangsu
University and the animal experiments followed the Affiliated Kunshan Hospital of Jiangsu University guidelines. The rats were randomly divided into three groups according to their treatment: the control group was treated with isotonic sodium chloride at the first and second surgery, the ORC group was treated with ORC at the first and second surgery, and the medical sodium hyaluronate (MSH) group was treated with MSH at the first and second surgery. All surgeries were performed under general anesthesia, and all efforts were made to minimize suffering.

\section{Surgical technique}

The animals were anesthetized with intraperitoneal injection of $100 \mathrm{mg} / \mathrm{L}$ chloral hydrate (300 mg/ $\mathrm{kg}$ body weight) and were then placed on the operating table in a supine position on a warm blanket. A $3 \mathrm{~cm}$ midline incision was made along the linea alba on the abdominal wall, and the abdominal cavity was opened to expose the uterus. At the corner of the uterus, a $1 \mathrm{~cm}$ incision was made until hemorrhage points appeared. The incision was then closed with 3-0 Dexon sutures, and one of the following agents was retained in the abdominal cavity: isotonic sodium chloride $(10 \mathrm{~mL})$ was used in the control group, ORC $(1.5 \times 1 \mathrm{~cm})$ was used in the ORC group, and $1 \% \mathrm{MSH}(0.5 \mathrm{~mL})$ was used in the MSH group. The abdominal wall was then closed (Figure 1). After surgery, the rats were exposed to infrared light until they awoke and were provided access to water alone for the first 6 hours. After a recovery period of 2 weeks, the rats underwent the second surgery and adhesiolysis by the same surgeon who was blinded to animal allocation, and the same agents were applied in the abdominal cavity of the rats (Figure 2A-D). Two weeks after the second surgery, a U-shaped incision was made to reveal the condition of intraperitoneal adhesions (Figure 2E, F). Finally, all rats were euthanized and the tissues in the adhesion zones were sent for pathological examinations.
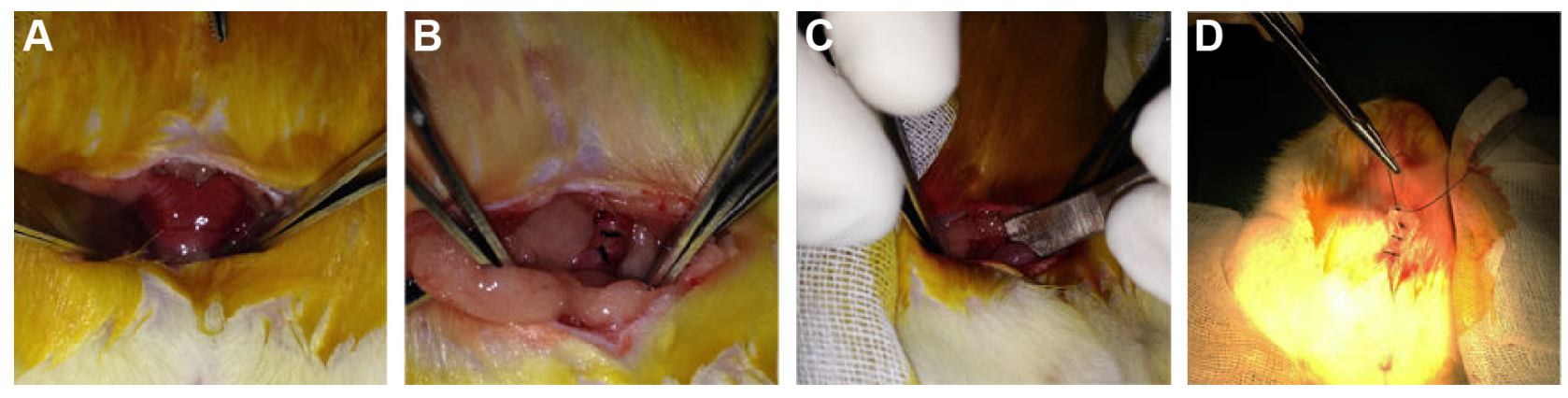

Figure I The surgical technique for creating the adhesion model.

Notes: (A) After a $3 \mathrm{~cm}$ incision was made, the uterus was externalized; (B) the uterus was incised and then sutured; (C) an agent (ORC) was then injected into the abdominal cavity; (D) the abdominal wall was closed. 

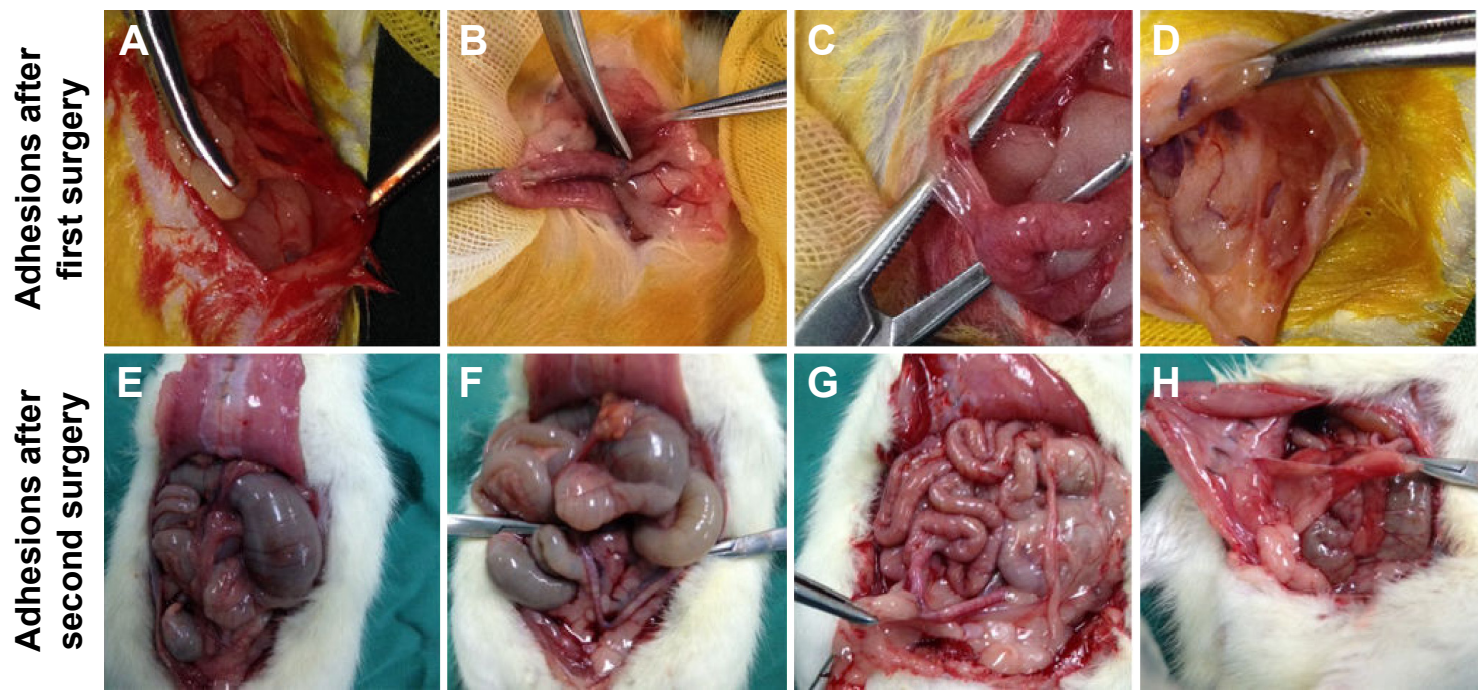

Figure 2 The representative adhesions after the first surgery $(\mathbf{A}-\mathbf{D})$ and second surgery $(\mathbf{E}-\mathbf{H})$.

Notes: (A) The adhering zone between the gastrocolic omentum and the abdominal wall; (B) the adhering zone between the gastrocolic omentum and the uterus; (C) the adhering zone between the uterus and the abdominal wall; (D) complicated and tight adhesions or internal organs sticking to the abdominal wall; (E) no adhesion; (F) the adhering zone between the gastrocolic omentum and the uterus; $(\mathbf{G})$ the adhering zone between the gastrocolic omentum and the uterus; $(\mathbf{H})$ the adhering zone between the gastrocolic omentum and the abdominal wall.

\section{Evaluation of postoperative adhesion}

The severity of postoperative adhesions was evaluated according to a modified Niar's scoring system as follows: ${ }^{12}$ 0 = no adhesion;

$1=$ one adhering zone between internal organs or between internal organs and the abdominal wall, equaling $1 \%-25 \%$ of uterine involvement;

$2=$ two adhering zones between internal organs or between internal organs and the abdominal wall, equaling $26 \%-50 \%$ of uterine involvement;

$3=$ more than two adhering zones between internal organs or between internal organs and the abdominal wall, which equals to $51 \%-75 \%$ of uterine involvement;

4 = complicated and close-up adhesions or direct attachment of internal organs to the abdominal wall irrespective of the number and circumscription of the adhering zones, which equals to $76 \%-100 \%$ of uterine involvement.

Adhesion scorings were evaluated by two investigators blinded to the treatment regimen. In addition, the body weight of each rat was measured every week during the experiment.

\section{Statistical analyses}

All measurement data and ranked data are reported as mean \pm standard deviation (SD). Student's $t$-tests were performed to identify the significant differences between two independent groups. Adhesion scores and weights of multiple groups were established by analysis of variance (ANOVA). Data regarding adhesion scoring were analyzed and compared using the Kruskal-Wallis test, and the Mann-Whitney $U$-test was utilized for intergroup dual comparisons if any statistical significance was found. A $P$-value of $<0.05$ was considered as statistically significant. All analyses were performed with SPSS 17.0 software (SPSS Inc., Chicago, IL, USA).

\section{Results}

\section{Adhesion evaluation}

The adhesions in the ORC and MSH groups were milder compared to the control group at 14 days after first surgery. In the ORC and MSH groups, adhesions were generally loose and consistent, and most were of the omental type, which could be easily separated by blunt dissection. However, in the control group, the adhesions were much stronger, and some adhesions could only be separated by sharp dissection; appearance of clear vascularization was also observed in complicated and tight adhesions or where internal organs stuck to abdominal wall. Fourteen days after the second surgery, the adhesions in the ORC and MSH groups showed no further aggravation. However, adhesions in the control group continued to worsen. Most adhesions could only be separated by sharp dissection resulting in visceral damage. The adhesion scores were calculated as follows: $3.00 \pm 0.82$ in the control group, $1.90 \pm 0.99$ in the $\mathrm{MSH}$ group, and $1.40 \pm 0.97$ in the ORC group at 2 weeks after the first surgery and 3.50 \pm 0.53 in the control group, $2.00 \pm 0.82$ in the MSH group, and $1.50 \pm 1.27$ in the ORC group at 2 weeks after the second surgery (Figure 3). The MSH and ORC groups had 


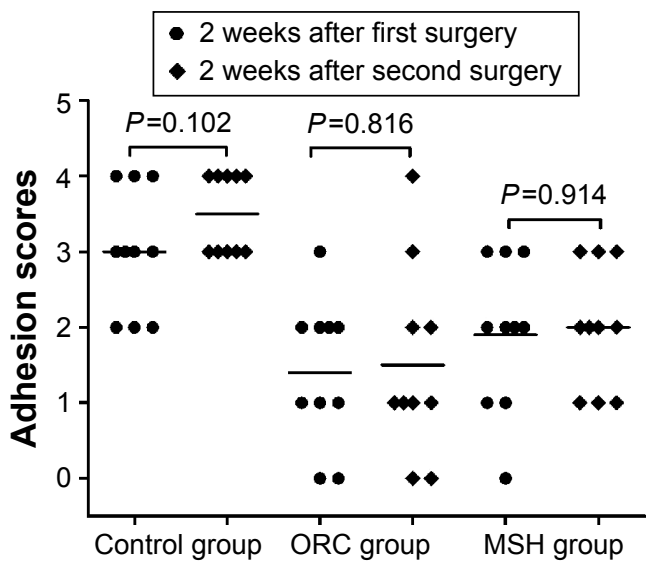

Figure 3 The adhesion scores of the control, ORC, and MSH groups at 2 weeks after first or second surgery.

Abbreviations: ORC, oxidized regenerated cellulose; $\mathrm{MSH}$, medical sodium hyaluronate.

significantly lower adhesion scores than the control group at day $14(P=0.005)$ and at day $28(P=0.001)$. No significant difference was found among the control group $(P=0.102)$, the MSH group $(P=0.914)$, or the ORC group $(P=0.816)$ with respect to adhesion scores at 2 weeks after the first surgery and at 2 weeks after the second surgery.

\section{Changes in body weight}

No mortality was observed during this study. The body weights of all 30 Sprague Dawley rats were measured before the first surgery and were measured once a week thereafter until they were sacrificed. The day of the time of first surgery was set as day 1 to form the timeline. The changes in body weight are depicted in Figure 4. At the beginning of

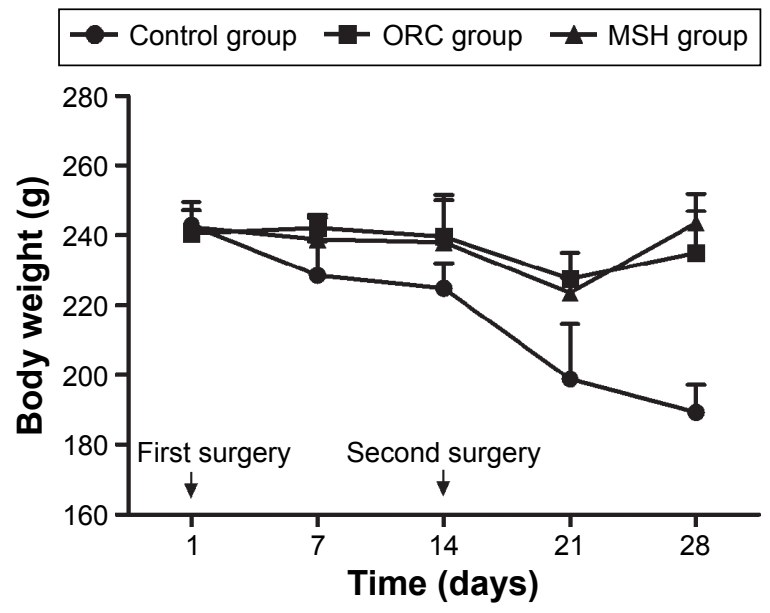

Figure 4 The body weights in each group are depicted.

Notes: At day I, the body weights in the control, MSH, and ORC groups were $242.8 \pm 6.8,242.3 \pm 5.0$, and $240.5 \pm 6.6$ g, respectively. On day 14 , the body weights in

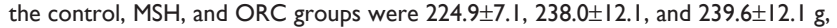
respectively. On day 28 , the body weights in the control, ORC, and MSH groups were 189.3 $\pm 7.8,245.5 \pm 9.0$, and $241.9 \pm 6.7 \mathrm{~g}$, respectively.

Abbreviations: ORC, oxidized regenerated cellulose; $\mathrm{MSH}$, medical sodium hyaluronate. the experiment, there was no significant difference in body weights between the three groups $(P=0.688)$. At day 14 , the body weights of rats were significantly different between the three groups $(P=0.009)$. The weight of rats in the control group was lower than that in the $\mathrm{MSH}(P=0.011)$ and ORC groups $(P=0.005)$ on day 14 . On day 28 , the weights were significantly higher in both the $\mathrm{MSH}$ and $\mathrm{ORC}$ groups compared to the control group $(P=0.000)$. Notably, the weight in the control group showed a consistent decrease on day 14 and day 28 compared to that before the first surgery $(P=0.000)$. On the other hand, the body weights were not changed significantly during these 2 weeks in both the MSH $(P=0.207)$ and ORC $(P=0.843)$ groups.

\section{Histological examination}

In order to investigate the pathogenesis of adhesion formation/ reformation, we performed histological evaluation of the adhesions on the uterus-abdominal wall and omentum-abdominal wall. Two weeks after the first surgery, tissue staining showed obvious proliferation of fibrous tissue, low-level inflammatory infiltration, and foreign body giant cells around the suture knot in the uterus-abdominal wall (Figure 5A). At 2 weeks after the second surgery, a similar histological change appeared in the adhesion reformation of the uterus-abdominal wall with a higher level of inflammatory infiltration, fibrous tissue growth around vessels, and initiation of blood vessel ingrowth (Figure 5C). In the omentum-abdominal wall, at 2 weeks after the first surgery, tissue staining showed proliferation of fibrous tissue, formation of granulation tissue, a low level of inflammatory infiltration, and foreign body giant cell (Figure 5B). At 2 weeks after the second surgery, tissue staining showed proliferation of fibrous tissue and adipose tissue along with local angiectasis and hemorrhage with inflammatory infiltration was observed (Figure 5D).

\section{Discussion}

Postsurgical adhesion is a complicated process and is mainly formed by an imbalance between fibrogenesis and fibrinolysis induced by infection and surgical trauma. ${ }^{1,13}$ The adhesions usually develop in the peritoneal membrane within 4-6 days of the surgery and are an inevitable problem for any surgeon. ${ }^{3}$ Decreased fibrinolytic activity and gain in fibrogenesis contribute to adhesion formation. ${ }^{14,15}$ Hypoxia plays a central role in the formation of adhesions. Hypoxia leads to oxidative stress, anaerobic metabolism, formation of free radicals, and eventual adhesion formation. The high levels of free radicals initiate a cascade of inflammatory pathways. ${ }^{16,17}$ Many cellular and humoral factors affect the adhesion formation by influencing the process of fibrogenesis 


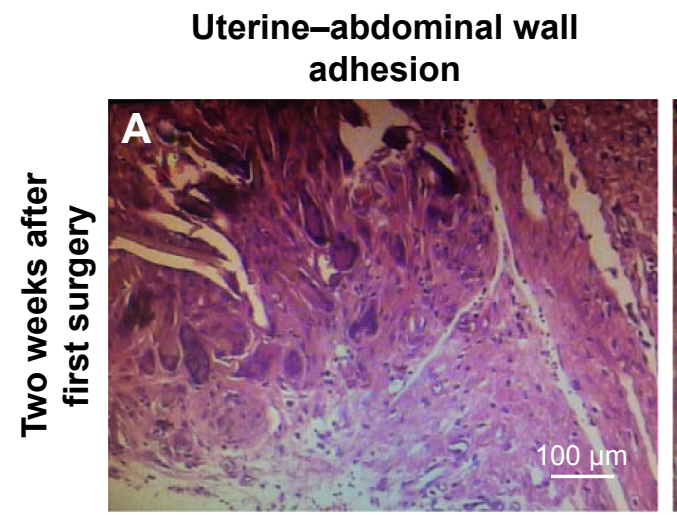

\section{Omentum-abdominal wall adhesion}
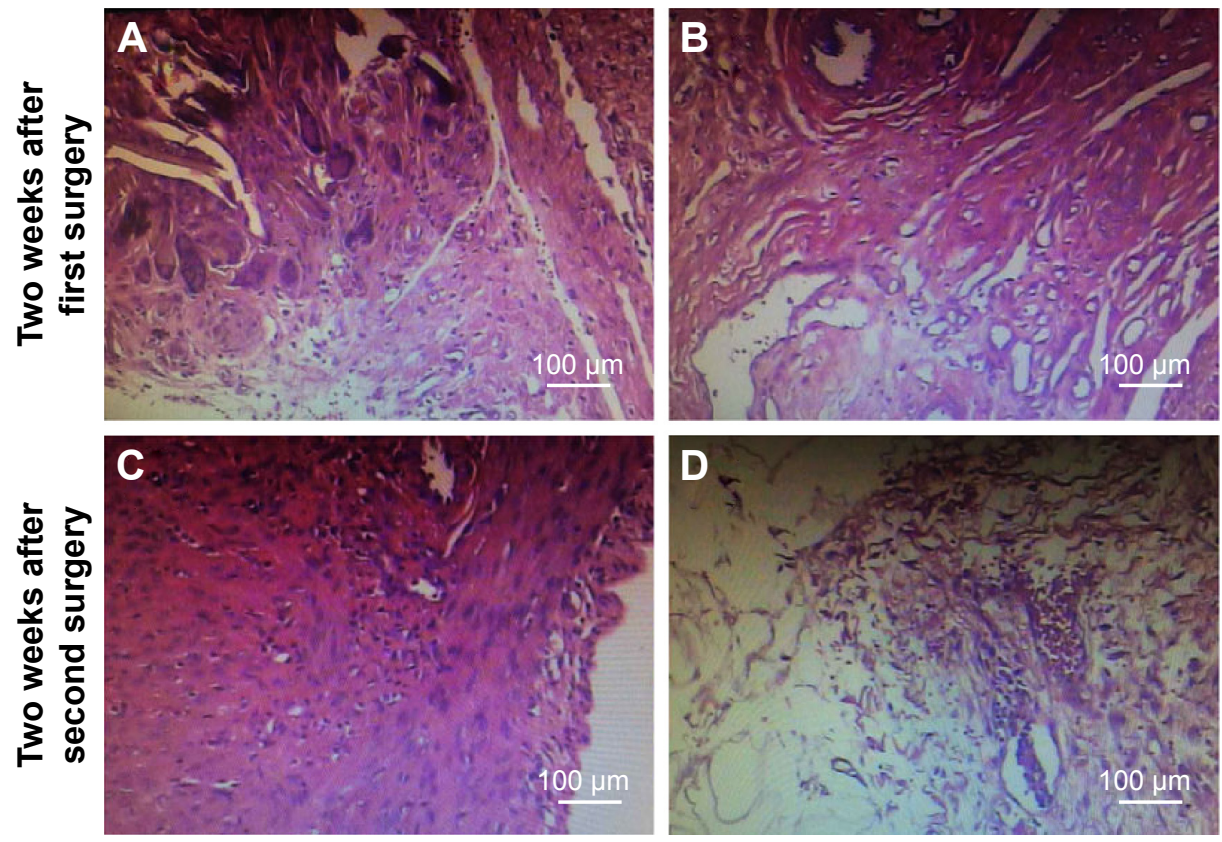

Figure 5 Hematoxylin-eosin staining of representative adhesion formation.

Notes: Adhesion formation in the uterus-abdominal wall and the omentum-abdominal wall at 2 weeks after the first surgery (A, B) and adhesion reformation at 2 weeks after the second surgery (C, D) are depicted, (I00X).

and fibrinolysis, including transforming growth factor- $\beta 1$, vascular endothelial growth factor, and tumor necrosis factor-alpha. ${ }^{16,18}$ Several types of inflammatory cells including granulocytes, lymphocytes, macrophages, and fibroblasts actively participate in the process of adhesion formation. ${ }^{18}$ Our results revealed the inflammatory response involved in adhesion formation/reformation (Figure 5). Besides, since physical contact is necessary, separation of the peritoneal tissue and the adjacent organs may be effective in preventing both adhesion formation and reformation. ${ }^{19}$

Based on the mechanism of adhesion formation, antiadhesion agents, including pharmacological agents and nonpharmacological agents developed by targeting activation of fibrinolysis, hampering of fibrogenesis, downregulation of inflammatory response, and inhibition of collagen synthesis have been investigated. ${ }^{1,11}$ At present, even though several agents such as tadalafil, ${ }^{10}$ melatonin, and dexamethasone ${ }^{11,14,20}$ are tested in animals and proven to be effective for preventing adhesion formation/reformation, no pharmacological agents are available for humans. Therefore, numerous studies are now focused on nonpharmacological agents to prevent adhesions. Nonpharmacological anti-adhesive agents are available in many forms, including liquid, membrane, and textile embodiments, and they mainly provide a physical barrier between adjacent peritoneal surfaces and organs, thus reducing adhesion formation/reformation in clinical use. ${ }^{21}$
ORC and hyaluronic acid are frequently used anti-adhesive agents in the clinic.

ORC was the first generation of mechanical barrier agents used to cover the peritoneum, and has been approved by the US Food and Drug Administration (FDA) for prevention of adhesion development. ${ }^{4,16}$ ORC is composed of ORC fabric that provides a continuous coat over damaged tissues within 8 hours and will be completely absorbed within 2 weeks. ${ }^{15,22}$ In this study, ORC could significantly reduce adhesion formation and reformation compared to the control group in an experimental rat model. Similar to ORC, MSH also has no significant impact on the body weights of these rat models. In contrast, the body weights in the control group showed a gradual decrease. Although some studies have observed change in body weight, few studies have performed an extensive investigation..$^{23,24}$ This outcome may be derived from the peritoneal adhesions and their relevant complications, such as bowel obstruction and abdominal pain. ${ }^{25,26}$ Since the models used in our study were adult rats, their bodies would not show extreme changes under normal conditions. The unchanging body weights in the treated groups indicate that the anti-adhesive agents were conducive to postoperative recovery and helped avoid malnutrition. The present study demonstrated that oxidized regenerated cellulose could play an important role in reducing postsurgical de novo adhesions and reformation. A classical literature reported in literature 
found that ORC could reduce adhesion formation and significantly prevent adhesion reformation in rabbit and dog models. ${ }^{27}$ Recently, a systematic review demonstrated that ORC could reduce the incidence of both de-novo adhesions, as well as the reformation of adhesions. ${ }^{4}$ More importantly, ORC has no effects on either pain or fertility outcomes in women of reproductive age. ${ }^{4}$

Hyaluronic acid has excellent physical and biological features as an anti-adhesive agent. This high-density polymer shows high viscosity, viscoelasticity, and water-retaining capacity, and could therefore function as a lubricant. ${ }^{18}$ Additionally, hyaluronic acid is biocompatible, biodegradable (completely degrades within 5 days), and nontoxic, which enhances its popularity as a barrier agent. ${ }^{18,21}$ In earlier studies, hyaluronic acid was not found to reduce adhesion formation or reformation. ${ }^{28,29}$ However, with the development of basic research and synthesis technologies, more studies have demonstrated its role in preventing adhesion formation/reformation. In 1997, Rodgers et $\mathrm{al}^{30}$ demonstrated that administration of hyaluronic acid at the end of surgery could reduce adhesion formation in a standard rabbit model. Two years later, Osada et al found that $1 \%$ sodium hyaluronate could decrease the area of adhesion reformation in a rabbit model. ${ }^{31}$ However, relevant studies on rat models are relatively few. In this study, hyaluronic acid proved to significantly inhibit the adhesion formation/reformation in a rat model. These plausible results contribute to the excellent physical and biological features of hyaluronic acid. Thus, hyaluronic acid and various hyaluronic acid combinations show promise for providing efficient antiadhesive treatments in the future. ${ }^{32-34}$

Women undergoing gynecological surgery are exposed to a high risk of adhesion formation especially in ovarian surgery, endometriosis surgery, tubal surgery, myomectomy, and adhesiolysis. ${ }^{2}$ Although in adherence to microsurgical principles, minimally invasive surgery is widely accepted, it is not sufficient to prevent adhesion formation/reformation. ${ }^{16}$ Therefore, in addition to applying meticulous surgical techniques and principles of microsurgery, proper use of anti-adhesion agents could reduce the incidence of adhesion formation/reformation. ${ }^{1}$ In conclusion, hyaluronic acid and ORC proved to reduce adhesion scores and had no effect on body weights in the rat model. However, their feasibility and safety for preventing adhesion reformation in clinical practice still needs to be warranted.

\section{Acknowledgments}

The study was supported by Kunshan Science and Technology Project (NO KS1349).

\section{Disclosure}

The authors report no conflicts of interest in this work.

\section{References}

1. Hirschelmann A, Tchartchian G, Wallwiener M, et al. A review of the problematic adhesion prophylaxis in gynaecological surgery. Arch Gynecol Obstet. 2012;285(4):1089-1097.

2. De Wilde RL, Brolmann H, Koninckx PR, et al. Prevention of adhesions in gynaecological surgery: the 2012 European field guideline. Gynecol Surg. 2012;9(4):365-368.

3. Wallwiener M, Brucker S, Hierlemann H, et al. Innovative barriers for peritoneal adhesion prevention: liquid or solid? A rat uterine horn model. Fertil Steril. 2006;86(Suppl 4):1266-1276.

4. Ahmad G, O'Flynn H, Hindocha A, et al. Barrier agents for adhesion prevention after gynaecological surgery. Cochrane Database Syst Rev. 2015;4:CD000475

5. Ergul E, Korukluoglu B. Peritoneal adhesions: facing the enemy. Int $J$ Surg. 2008;6(3):253-260.

6. Johns DB, Keyport GM, Hoehler F, et al. Reduction of postsurgical adhesions with Intergel adhesion prevention solution: a multicenter study of safety and efficacy after conservative gynecologic surgery. Fertil Steril. 2001;76(3):595-604.

7. Bristow RE, Montz FJ. Prevention of adhesion formation after radical oophorectomy using a sodium hyaluronate-carboxymethylcellulose (HA-CMC) barrier. Gynecol Oncol. 2005;99(2):301-308.

8. Brown CB, Luciano AA, Martin D, et al. Adept (icodextrin $4 \%$ solution) reduces adhesions after laparoscopic surgery for adhesiolysis: a double-blind, randomized, controlled study. Fertil Steril. 2007;88(5):1413-1426.

9. Hindocha A, Beere L, Dias S, et al. Adhesion prevention agents for gynaecological surgery: an overview of Cochrane reviews. Cochrane Database Syst Rev. 2015;1:CD011254.

10. Kutuk MS, Ozgun MT, Batukan C, et al. Oral tadalafil reduces intraabdominal adhesion reformation in rats. Hum Reprod. 2012;27(3): 733-737.

11. Oner G, Ulug P. A systemic review of randomized controlled studies about prevention with pharmacologic agents of adhesion formation in the rat uterine horn model. Arch Med Sci. 2015;11(2):274-281.

12. Nair SK, Bhat IK, Aurora AL. Role of proteolytic enzyme in the prevention of postoperative intraperitoneal adhesions. Arch Surg. 1974; 108(6):849-853.

13. Hellebrekers BW, Trimbos-Kemper TC, Trimbos JB, et al. Use of fibrinolytic agents in the prevention of postoperative adhesion formation. Fertil Steril. 2000;74(2):203-212.

14. Attar R, Yildirim G, Kumbak B, et al. Efficacy of melatonin and hyaluronate/carboxymethylcellulose membrane in preventing adhesion reformation following adhesiolysis in a rat uterine model. JObstet Gynaecol Res. 2011;37(2):125-131.

15. Practice Committee of the American Society for Reproductive Medicine. Control and prevention of peritoneal adhesions in gynecologic surgery. Fertil Steril. 2006;86(5 Suppl 1):S1-S5.

16. Braun KM, Diamond MP. The biology of adhesion formation in the peritoneal cavity. Semin Pediatr Surg. 2014;23(6):336-343.

17. Wei G, Chen X, Wang G, et al. Inhibition of cyclooxygenase-2 prevents intra-abdominal adhesions by decreasing activity of peritoneal fibroblasts. Drug Des Devel Ther. 2015;9:3083-3098.

18. Brochhausen C, Schmitt VH, Rajab TK, et al. Intraperitoneal adhesions - an ongoing challenge between biomedical engineering and the life sciences. J Biomed Mater Res A. 2011;98(1):143-156.

19. Kamel RM. Prevention of postoperative peritoneal adhesions. Eur $J$ Obstet Gynecol Reprod Biol. 2010;150(2):111-118.

20. Du XH, Liu JQ, Xin K, et al. Dexamethasone and sodium carboxymethyl cellulose prevent postoperative intraperitoneal adhesions in rats. Braz J Med Biol Res. 2015;48(4):344-348.

21. Back JH, Cho WJ, Kim JH, et al. Application of hyaluronic acid/sodium alginate-based microparticles to prevent tissue adhesion in a rabbit model. Surg Today. 2016;46(4):501-508. 
22. Liakakos T, Thomakos N, Fine PM, et al. Peritoneal adhesions: etiology, pathophysiology, and clinical significance. Recent advances in prevention and management. Dig Surg. 2001;18(4):260-273.

23. Ito T, Yeo Y, Highley CB, et al. Dextran-based in situ cross-linked injectable hydrogels to prevent peritoneal adhesions. Biomaterials. 2007; 28(23):3418-3426.

24. Yeo Y, Adil M, Bellas E, et al. Prevention of peritoneal adhesions with an in situ cross-linkable hyaluronan hydrogel delivering budesonide. J Control Release. 2007;120(3):178-185.

25. Practice Committee of the American Society for Reproductive Medicine; Society of Reproductive Surgeons. Pathogenesis, consequences, and control of peritoneal adhesions in gynecologic surgery. Fertil Steril. 2007;88(1):21-26.

26. Izumi Y, Yamamoto M, Kawamura M, et al. Cross-linked poly (gammaglutamic acid) attenuates peritoneal adhesion in a rat model. Surgery. 2007;141(5):678-681.

27. Shimanuki T, Nishimura K, Montz FJ, et al. Localized prevention of postsurgical adhesion formation and reformation with oxidized regenerated cellulose. J Biomed Mater Res. 1987;21(2):173-185.

28. Urman B, Gomel V. Effect of hyaluronic acid on postoperative intraperitoneal adhesion formation and reformation in the rat model. Fertil Steril. 1991;56(3):568-570.
29. West JL, Chowdhury SM, Sawhney AS, et al. Efficacy of adhesion barriers. Resorbable hydrogel, oxidized regenerated cellulose and hyaluronic acid. J Reprod Med. 1996;41(3):149-154.

30. Rodgers K E, Johns D B, Girgis W, et al. Reduction of adhesion formation with hyaluronic acid after peritoneal surgery in rabbits[J]. Fertil Steril. 1997;67(3):553-558.

31. Osada H, Takahashi K, Fujii TK, et al. The effect of cross-linked hyaluronate hydrogel on the reduction of post-surgical adhesion reformation in rabbits. J Int Med Res. 1999;27(5):233-241.

32. Kaya C, Sever N, Cengiz H, et al. A randomized controlled study of the efficacy of misoprostol and hyaluronic acid in preventing adhesion formation after gynecological surgery: a rat uterine horn model. Eur $J$ Obstet Gynecol Reprod Biol. 2014;176:44-49.

33. ten Broek RP, Stommel MW, Strik C, et al. Benefits and harms of adhesion barriers for abdominal surgery: a systematic review and meta-analysis. Lancet. 2014;383(9911):48-59.

34. Pados G, Venetis CA, Almaloglou K, et al. Prevention of intra-peritoneal adhesions in gynaecological surgery: theory and evidence. Reprod Biomed Online. 2010;21(3):290-303.

\section{Publish your work in this journal}

Drug Design, Development and Therapy is an international, peerreviewed open-access journal that spans the spectrum of drug design and development through to clinical applications. Clinical outcomes, patient safety, and programs for the development and effective, safe, and sustained use of medicines are the features of the journal, which has also been accepted for indexing on PubMed Central. The manuscript management system is completely online and includes a very quick and fair peer-review system, which is all easy to use. Visit http://www.dovepress.com/testimonials.php to read real quotes from published authors.

Submit your manuscript here: http://www.dovepress.com/drug-design-development-and-therapy-journal 